\title{
Diffusion tensor imaging based multiparametric characterization of renal lesions in infants with urinary tract infections: an explorative study
}

Yvonne Simrén $^{1 *}$ D, Eira Stokland ${ }^{1}$, Sverker Hansson $^{2}$, Hanna Hebelka $^{1}$, Par-Arne Svensson $^{1}$ and Kerstin M. Lagerstrand ${ }^{3}$

\begin{abstract}
Background: Conventional diffusion weighted imaging (DWI) is a promising non-invasive tool in the evaluation of infants with symptomatic urinary tract infections (UTI). The use of multiparametric diffusion tensor imaging (DTI) provides further information on renal pathology by reflecting renal microstructure. However, its potential to characterize and distinguish between renal lesions, such as acute pyelonephritic lesions, permanent renal damages or dysplastic changes has not been shown. This study aimed to evaluate the potential of multiparametric DTI for characterization of renal lesions with purpose to distinguish acute pyelonephritis from other renal lesions in young infants with their first UTI.
\end{abstract}

Methods: Nine kidneys in seven infants, age 1.0-5.6 months, with renal lesions i.e. uptake reductions, on acute scintigraphy performed after their first UTI, were included. The DTI examinations were performed during free breathing without sedation. The signal in the lesions and in normal renal tissue was measured in the following images: b0, b700, apparent diffusion coefficient (ADC), and fractional anisotropy (FA). In addition, DTI tractographies were produced for visibility.

Results: There was a difference between lesions and normal tissue in b700 signal (197 \pm 52 and $164 \pm 53, p=0.011$ ), $\operatorname{ADC}\left(1.22 \pm 0.11\right.$ and $\left.1.45 \pm 0.15 \mathrm{~mm}^{2} / \mathrm{s}, p=0.008\right)$, and FA $(0.18 \pm 0.03$ and $0.30 \pm 0.10, p=0.008)$ for all nine kidneys. Six kidneys had focal lesions with increased b700 signal, decreased ADC and FA indicating acute inflammation. In three patients, the multiparametric characteristics of the lesions were diverging.

Conclusion: Multiparametric DTI has the potential to further characterize and distinguish acute pyelonephritis from other renal lesions in infants with symptomatic UTI.

Keywords: Diffusion tensor imaging, Infant, Kidney, Pyelonephritis, Urinary tract infection

\footnotetext{
* Correspondence: yvonne.simren@gu.se

'Department of Radiology, Institute of Clinical Sciences at Sahlgrenska Academy, University of Gothenburg, Gothenburg, Sweden

Full list of author information is available at the end of the article
}

(c) The Author(s). 2021 Open Access This article is licensed under a Creative Commons Attribution 4.0 International License, which permits use, sharing, adaptation, distribution and reproduction in any medium or format, as long as you give appropriate credit to the original author(s) and the source, provide a link to the Creative Commons licence, and indicate if changes were made. The images or other third party material in this article are included in the article's Creative Commons licence, unless indicated otherwise in a credit line to the material. If material is not included in the article's Creative Commons licence and your intended use is not permitted by statutory regulation or exceeds the permitted use, you will need to obtain permission directly from the copyright holder. To view a copy of this licence, visit http://creativecommons.org/licenses/by/4.0/ The Creative Commons Public Domain Dedication waiver (http://creativecommons.org/publicdomain/zero/1.0/) applies to the data made available in this article, unless otherwise stated in a credit line to the data. 


\section{Background}

Urinary tract infection (UTI) is a common disease in infants with an incidence of $2 \%$ in children under 1 year of age [1]. Ultrasound is often used as a first line investigation of infants with UTI. The use of further imaging varies depending on which protocol that is used. When further imaging is needed, for example to evaluate the extent of engagement of the renal tissue or to identify complications of the infection, dependent on availability, 99mTc-Dimercaptosuccinic acid (DMSA) scintigraphy, computed tomography (CT) or contrast enhanced magnetic resonance imaging (MRI) can be used. However, DMSA scintigraphy and contrast-enhanced techniques are invasive as they require an intravenous injection. DMSA scintigraphy and CT also has the drawback of using ionizing radiation. Therefore, diffusion weighted imaging (DWI), a non-invasive MRI-technique, has been suggested as an alternative method in the evaluation of children with symptomatic UTI [2-6].

Renal parenchymal lesions detected in imaging of younger infants with an UTI may have different etiologies, such as acute inflammation i.e. acute pyelonephritis, permanent renal damage or dysplastic changes that are important to differentiate as it will impact the clinical management of the patient. DWI in multiple direction, i.e. diffusion tensor imaging (DTI), can give an insight into the microstructure of the renal tissue as the use of multiple diffusion directions better depicts the highly radiating oriented renal structure [7-12]. This technique enables calculation of several quantitative measures that can be used in the diagnosis together with the findings from the visual evaluation. The comprehensive analysis of multiple DTI parameters i.e. multiparametric DTI, can provide further information that may improve the characterization of renal lesions in infants with symptomatic UTI. Recent studies have shown the potential of the technique to evaluate renal pathology in older children [13-17]. It has also been shown that free breathing DTI is feasible and can generate images with higher quality compared to conventional DWI in children with acute pyelonephritis [18]. To our knowledge, there are no studies on renal DTI in young infants, a patient group that would benefit from the use of this noninvasive, non-radiating, short scan technique. The potential of DWI for detection of acute pyelonephritis has recently been shown in non-sedated free breathing infants with symptomatic UTI [6]. However, in several infants' lesions the findings were equivocal and not typical for acute pyelonephritis raising the need for further characterization of such lesions.

Aim: To study the potential of multiparametric DTI for characterization of renal lesions with purpose to distinguish different renal lesions in young infants with their first UTI.

\section{Methods}

\section{Patients}

This explorative study was performed in conjunction with a recently published study [6] including infants (< 6 months of age) that had been examined with acute DMSA scintigraphy as part of the clinical routine follow-up of infants with first time community-acquired symptomatic UTI in our hospital over a period of 19 months. The MRI examinations were performed within two weeks of the acute DMSA scintigraphy. Fourteen patients with uptake reductions on the DMSA images were eligible for the study. Seven patients were included as they had DWI as well as DMSA images of high diagnostic quality without artefacts.

Written informed consent for participation in this study was obtained from the guardians of the children. The study was approved by the Swedish Ethical Review Authority in Gothenburg, Gothenburg, Sweden (Dnr 214-11).

\section{Imaging methods}

The MRI examination was performed in median 1 day (range 0-12 days) from onset of the infection and 1 day from the acute DMSA examination (range 0-7 days). All infants underwent MRI examinations, sleeping or resting in the scanner after feeding, without any use of sedation or anesthetics. The scanning was performed during free breathing using a $1.5 \mathrm{~T}$ MRI system (GE Signa HDx, Twinspeed, GE Medical systems, Waukesha, WI, USA) with a $40 \mathrm{mT} / \mathrm{m}$ maximum gradient capability using the body coil as radiofrequency transmitter and the eightchannel cardiac coil as radiofrequency receiver. The total examination time was $15 \mathrm{~min}$, with an effective acquisition time of under $5 \mathrm{~min}$, including a prescan and a T2 weighted scan for verification of morphological findings and volume measurements. The acquisition time of the DTI scan was $58 \mathrm{~s}$.

The DTI scanning was performed using coronal and fat saturated single-shot spin-echo planar 2D imaging, $\mathrm{TR}=2000 \mathrm{~ms}, \quad \mathrm{TE}=66 \mathrm{~ms}$, acceleration factor $=2$, bvalue $=0$ and $700 \mathrm{~s} / \mathrm{mm}^{2}, \mathrm{NEX}=1,24$ diffusion directions. Ten coronal slices with a thickness of $6 \mathrm{~mm}$ and a scan pixel size of $2.2 \times 1.8 \mathrm{~mm}^{2}$ were used to cover both kidneys. For all infants, saturation bands were used to suppress regions with moving structures and thereby reduce the appearance of artifacts in the DWI scans. For calculation of b700, all diffusion directions from the b700 s/mm $\mathrm{m}^{2}$ acquisitions were used. Apparent diffusion coefficient (ADC) maps were calculated using a monoexponential model. DTI, ADC, fractional anisotropy (FA) maps and tractographies were reconstructed on the workstation of the MRI system (Advantage Workstation, GE Healthcare, USA). The tractographies were launched from seeds within a ROI including the whole kidney on 
the coronal slice with findings on the b700 image. The FA threshold (0.05-0.1) and the maximum angle threshold $\left(55^{\circ}-100^{\circ}\right)$ for the tractography were chosen to get high density of tracks with limited spurious tracks outside the boundaries of the kidneys [13].

The DMSA examinations were performed in accordance with the guidelines of the Pediatric Committee of the European Association of Nuclear Medicine [19]. All DMSA images were reviewed by a senior nuclear medicine specialist. The criterion for lesions on the DMSA images was the presence of uptake reductions. Followup DMSA examinations were performed 1 year after the first DMSA examination as part of the clinical routine.

\section{Image analyses}

The descriptive image evaluation was performed on a diagnostic workstation with adjustment of the optimal window setting on a case-by-case basis. Two senior pediatric radiologists reviewed the images in consensus. The image sets were evaluated with regard to signal changes in the renal parenchyma corresponding to the lesions detected on the acute DMSA images. The images were also evaluated for any morphological findings such as hydronephrosis or duplications. The pattern of the tracks from the tractography was evaluated for any presence of distortion or reduction of tracks.

Quantitative image analyses were performed by a senior pediatric radiologist using Image $\mathrm{J}(1.45 \mathrm{~s}$, National Institute of Health, USA). Renal volume estimation was performed after manually outlining the renal contour on all sliced on the T2 weighted images. For estimation of quantitative parameters, circular regions of interest (ROI), with an area of $50 \mathrm{~mm}^{2}$, were manually placed on the DTI images, as well as on the ADC and FA maps. All ROIs were positioned to avoid large vessels, the renal calyces, or renal pelvis. The lesions were evaluated with three ROIs placed inside the centre of the lesions using the acute DMSA images as a reference to verify the location. For the evaluation of normal renal tissue in unilateral lesions, ROIs were placed in both the apical, lateral and the caudal part of the contralateral kidney in. In patients with bilateral lesions, three ROIs were instead placed in areas of the same kidney where there were no uptake reductions on the DMSA scans or signal changes on the DTI images. The mean value of the three ROIs were used for further analyses. The multiparametric analysis was performed by inspecting the combined data from the descriptive and quantitative analyses, with the intention to find patterns among the characteristics of the different lesions.

\section{Statistical analyses}

The statistical analyses were performed in IBM SPSS Statistics 26 software package. For comparison of values between lesions and normal renal tissue, the Wilcoxon signed rank test was used and $p$-values of $<0.05$ were considered significant.

\section{Results}

Patients

DTI images from a total of seven infants, median age 1.2 months (range 1.0-5.6 months), were analysed. Two of the patients had bilateral lesions, resulting in a total of nine kidneys evaluated. Table 1 shows patient characteristics for the seven patients.

\section{Descriptive characteristics}

Table 2 shows the descriptive characteristics of the findings on the images for the different methods. The morphological evaluation revealed one patient (no 7) with thinner parenchyma in the apical part of the kidney, but no hydronephrosis, duplex or other malformations were found in any of the other kidneys. Focal increased signal on b700, corresponding to decreased $\mathrm{ADC}$ and distorted/less tracks on tractography, was seen in patient no 1, 2, 4 and 5. To exemplify the findings of this group, the images of patients no 2 and 4 are shown in Fig. 1.

In patients no 3, 6 and 7 (Fig. 2), the patterns were more diverse and differed regarding either the signal

Table 1 Patient characteristics for seven patients with renal lesions on the acute DMSA images

\begin{tabular}{llllllllll}
\hline Patient no & Sex & $\begin{array}{l}\text { Age } \\
\text { (months) }\end{array}$ & Bacteriuria & $\begin{array}{l}\text { Max temp } \\
\left({ }^{\circ} \mathbf{C}\right)\end{array}$ & $\begin{array}{l}\text { Max CRP } \\
(\mathbf{m g} / \mathbf{L})\end{array}$ & $\begin{array}{l}\text { Laterality of } \\
\text { renal lesion }\end{array}$ & $\begin{array}{l}\text { Vesico-ureteral } \\
\text { reflux grade } \\
\text { Right/Left }\end{array}$ & $\begin{array}{l}\text { Renal } \\
\text { volume (m) } \\
\text { Right/Left }\end{array}$ & $\begin{array}{l}\text { Follow-up } \\
\text { DMSA }\end{array}$ \\
\hline 1 & Boy & $>1.2$ & E. coli & 39.1 & 91 & Left & VCUG not performed & $28 / 35$ & Normal \\
2 & Girl & $\leq 1.2$ & E. coli & 38.7 & 44 & Left & $0 / 4$ & $18 / 17$ & Persistent lesions \\
3 & Boy & $>1.2$ & E. coli & 37.2 & 8 & Left & $0 / 0$ & $14 / 14$ & Normal \\
4 & Girl & $\leq 1.2$ & E. coli & 38.3 & 62 & Bilateral & $0 / 0$ & $23 / 22$ & Persistent lesions \\
5 & Boy & $\leq 1.2$ & E. coli & 38.6 & 147 & Bilateral & $0 / 0$ & $29 / 26$ & Normal \\
6 & Boy & $>1.2$ & E. coli & 40.3 & 90 & Left & $0 / 0$ & $18 / 20$ & Persistent lesions \\
7 & Girl & $\leq 1.2$ & Enterococci & 38.3 & 180 & Right & $2 / 0$ & $17 / 19$ & Persistent lesions \\
\hline
\end{tabular}

VCUG voiding cystourethrography, DMSA 99mTc-Dimercaptosuccinic acid scintigraphy 
Table 2 Descriptive characteristics of the findings on the images b0, b700, ADC and Tractography compared to normal tissue

\begin{tabular}{lllllll}
\hline Patient no & Laterality & DMSA uptake reduction & b0 signal & b700 signal & ADC value & Tractography tracks \\
\hline 1 & Left & Apical & Apical + & Apical + & Apical - & Distorted/Reduced \\
2 & Left & General & No change & General + & General - & Distorted/Reduced \\
3 & Left & Apical & No change & General + & General - & $\begin{array}{l}\text { Patchy distorted } \\
\text { pattern/Reduced }\end{array}$ \\
4 & Left & Apical & Apical + & Apical + & Apical - & Distorted/Reduced \\
4 & Right & Apical & No change & Apical + & Apical - & Distorted/Reduced \\
5 & Left & General & No change & Apical + & Apical - & Distorted/Reduced \\
5 & Right & Apical & No change & Apical + & Apical - & Distorted/Reduced \\
6 & Left & Apical & Apical - & Apical mix $+/-$ & Apical - & Distorted/Reduced \\
7 & Right & General & Apical - & No change & Apical - & Distorted/Reduced \\
\hline
\end{tabular}

DMSA 99mTc-dimercaptosuccinic acid scintigraphy, $A D C$ apparent diffusion coefficient

+ increase, - decrease

intensity on the different images or distribution of the findings.

\section{Quantitative characteristics}

Table 3 shows quantitative characteristics expressed as the difference in values between lesions and normal tissue. For patient no 6, the values were obtained from ROIs placed in the lesion but avoiding the small central area with reduced signal. There was a significant increase in signal between lesions and normal tissue for b700 (197 \pm 52 and $164 \pm 53, p=0.011)$ and corresponding decrease for ADC $\left(1.22 \pm 0.11\right.$ and $1.45 \pm 0.15 \mathrm{~mm}^{2} / \mathrm{s}$, $p=0.008)$ and FA $(0.18 \pm 0.03$ and $0.30 \pm 0.10, p=0.008)$. No significant difference in the b0 signal between lesions and normal tissue was found (460 \pm 91 and $458 \pm 153$, $p=0.375)$

\section{Multiparametric analyses}

All patients showed decreased FA and ADC value in lesions compared to normal tissue. In all patients, the decrease in FA was larger than the decrease in ADC. Kidney no 1, 2, 4 left, 4 right, 5 left, 5 right showed the pattern of focal lesions with distorted/less tracks on the descriptive analyses with corresponding quantitative findings of increased b700 signal, decreased ADC and FA.

Three patients differed from this pattern. Patient no 3 showed more generalized changes on the descriptive analysis and a larger difference between lesion and normal tissue for all three quantitative parameters compared to the other patients. In patient no 6 , the discrete signal changes that surrounded the small focal lesion followed the pattern of patient no 1, 2, 4 and 5 but the small focal lesion had low signal on b700. In contrast to

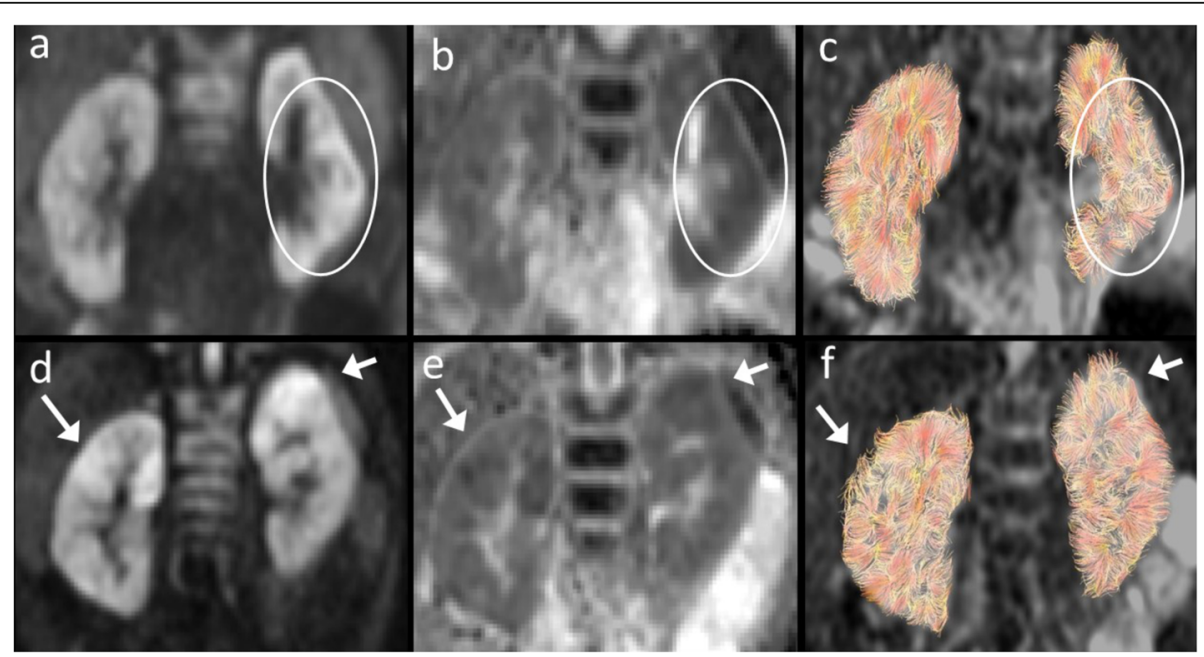

Fig. 1 Example of two infants, no $2 \mathbf{a}-\mathbf{c}$ and $4 \mathbf{d}-\mathbf{f}$, with lesions (circles/arrows) showing the characteristics of increased signal on the b700 a, d, reduced $A D C \mathbf{b}$, e and distorted/less tracks on tractographies $\mathbf{c}, \mathbf{f}$ 


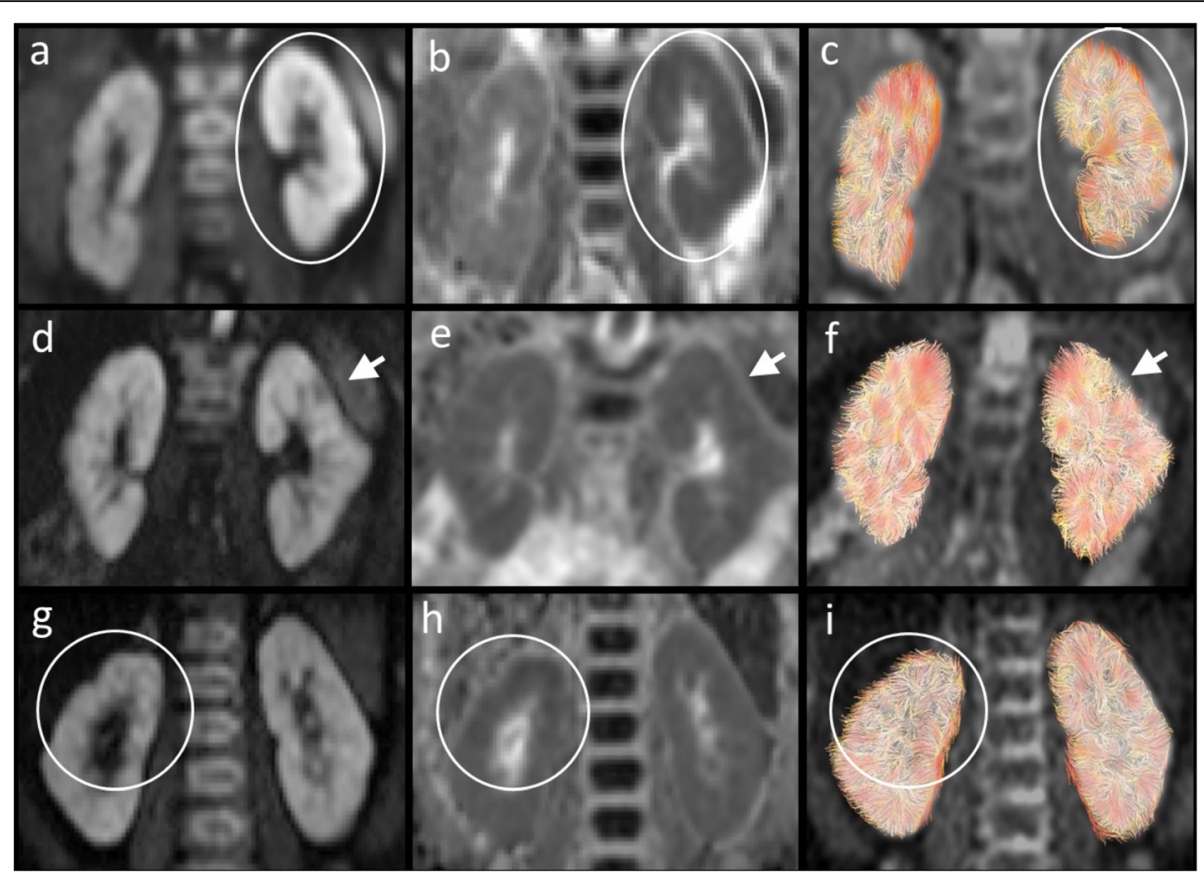

Fig. 2 Illustration of the patients (no 3, 6 and 7) with diverse DTI characteristics on the diffusion dependent images b700 a, d, g, ADC $\mathbf{b}, \mathbf{e}, \mathbf{h}$ and tractography $\mathbf{c}, \mathbf{f}, \mathbf{i}$. In patient no 3 (a-c) generalized changes were seen in the left kidney with increased b700, decreased ADC and patchy changes of the tracks on the tractography. In patient no $\mathbf{6} \mathbf{d}-\mathbf{f}$ there was an apico-lateral discrete increased signal with a central small low signal area (arrow) on the b700 of unclear etiologic. Distorted track pattern could be seen on the tractography in this area. In patient no $7 \mathbf{g}-\mathbf{i}$ there was a decreased ADC signal in the upper half of the right kidney showing less tracks with some distortion, but no signal increase could be depicted visually on the b700

all other patients, no 7 showed a decrease in ADC and FA but no corresponding increase in the b700 signal compared to normal tissue.

\section{Discussion}

This explorative study investigated the potential of multiparametric DTI in characterization of renal lesions in infants with their first UTI. The typical findings of acute pyelonephritis using conventional DWI has been described as areas of hindered diffusion [2-5]. The same findings have been reported using DTI [18]. Multiparametric DTI provides several parameters that can be used for further characterization of these findings. By combining these parameters, the lesion could be characterized further and lesion patterns were found. This complementary information may be valuable in clinical practise as it might be used to distinguish acute pyelonephritis or abscess from permanent renal damage or dysplastic changes.

In six of the nine kidneys, we found a pattern of focal hyperintense lesions on b700 with decreased ADC indicating hindered diffusion in the affected tissue. The lesions also showed alterations in anisotropy, confirmed

Table 3 Difference in b0, b700, ADC and FA in lesions relative normal renal tissue in nine kidneys in seven patients

\begin{tabular}{|c|c|c|c|c|c|}
\hline Patient no & Laterality & b0 signal diff & b700 signal diff & ADC diff & FA diff \\
\hline 1 & Left & $21 \%$ & $32 \%$ & $-12 \%$ & $-44 \%$ \\
\hline 2 & Left & $-2 \%$ & $26 \%$ & $-19 \%$ & $-35 \%$ \\
\hline 3 & Left & $3 \%$ & $38 \%$ & $-25 \%$ & $-56 \%$ \\
\hline 4 & Left & $21 \%$ & $33 \%$ & $-22 \%$ & $-35 \%$ \\
\hline 4 & Right & $11 \%$ & $24 \%$ & $-20 \%$ & $-38 \%$ \\
\hline 5 & Left & $0 \%$ & $22 \%$ & $-11 \%$ & $-34 \%$ \\
\hline 5 & Right & $8 \%$ & $25 \%$ & $-14 \%$ & $-38 \%$ \\
\hline 6 & Left & $-2 \%$ & $19 \%$ & $-7 \%$ & $-36 \%$ \\
\hline 7 & Right & $-23 \%$ & $-3 \%$ & $-13 \%$ & $-35 \%$ \\
\hline
\end{tabular}


by significant changes in FA. We think it is likely that this pattern is typical for acute pyelonephritis. There is limited knowledge on the pathophysiological mechanisms accounting for the abnormalities found in imaging of pyelonephritic changes but it is suggested to be multifactorial, with the inflammatory response causing oedema and accumulation of intravascular and intratubular granulocytes with secondary focal ischemia [20, 21]. We hypothesize that the findings in our study with diffusion restriction as well as the reduced FA could be explained by these inflammatory changes. The fact that the decrease in FA between the lesions and normal parenchyma was greater than the decrease in the ADC could imply that the highly radially oriented tubules in the kidney makes the FA more sensitive to cell infiltration in the tubules compared to ADC [8]. This would eventually also lead to loss of the normally radiating pattern that could be seen on the tractographies in these six kidneys. In concordance with our expectations, there was no significant difference in the b0 signal in the lesions compared to normal tissue.

In the other three patients (no 3, 6 and 7), the patterns differed from the group of patients with more typical pyelonephritic pattern. In patient no 3, more generalized changes in the parenchyma was seen and with a larger difference between lesion and normal tissue for all three quantitative parameters compared to the other patients. There was no increase in renal volume that indicated renal swelling and clinically the inflammatory parameters were low; thus, the nature of the finding is unclear. However, the etiology cannot be determined without histopathological correlation. In patient no 6 , the descriptive characteristic of the lesion was discrete hyperintensity that might have been overseen visually but showed typical pattern of acute pyelonephritis when evaluated further with quantitative measures. The central low signal area on the b700 was difficult to characterize, due to its small size but the signal pattern could suggest a minimal remnant abscess or bleeding. In contrast to the other patients, no 7 showed a decrease in ADC and FA in the lesion compared to normal parenchyma, but no corresponding increase in the b700 signal. This low diffusion signal (b700) can to some extent be the result of a very low T2 signal (observed on the b0 images) as the b700 signal reflects both the diffusion and the T2 signal in the tissue. The radial pattern on the tractography was also highly disturbed. The thin parenchyma in the affected part of the kidney, together with findings that were persistent on the follow-up of the patient, suggests that these changes most probably were of hypoplastic/dysplastic type. We suggest that the low b700 signal could indicate that there was no acute edema and that this lesion was in fact permanent.
Previous studies have shown that DTI is a valuable tool to evaluate renal pathology in the pediatric population [14-18]. Reports have suggested that DTI-based parameters, including ADC and FA, are potential biomarkers in the evaluation of kidneys with ureteropelvic junction obstruction $[14,16]$. DTI has also been described as a promising non-invasive method in the assessment of renal allograft health in pediatric allograft recipients [17]. There are a few reports on the potential of DTI to provide complementary information to DWI in assessment of various focal lesions in the adult and pediatric populations $[11,13,22]$ but the value of using DTI in the diagnosis of acute pyelonephritis is sparsely studied. Lair et al. studied 31 children (age 6 months of age to 16 years) with suspected acute pyelonephritis with the objective to compare image quality in free breathing DTI compared to respiratory triggered conventional DWI [18]. They found that DTI demonstrated significantly better image quality and showed agreement with DWI in all patients. Although the goal of the study was not to assess the diagnostic value of DTI, they concluded that DTI could replace DWI in the diagnosis of acute pyelonephritis.

The use of free breathing methods in children is attractive since MR examinations in children is demanding due to long scan times and a need for sedation. Infants under 6 month of age can often be scanned during sleep after feeding if an optimized scan protocol is used. In this study we used a short scan protocol that was optimized for non-sedated free breathing scanning with an acquisition time for the DTI scan of under $1 \mathrm{~min}$. This is a great advantage in younger infants and increases the clinical usability of the method. Moreover, the ability of MR methods to detect or rule out confounding pathologies, such as duplications or other malformations, is another strength and limits the need for further imaging.

However, the use of a scan protocol optimized for non-sedated free breathing scanning in combination with the thin cortex in young infants did not allow for adequate differentiation between the cortex and medulla for separate analyses. In addition, the small kidney size in younger infants makes the ROI placement in the lesions and normal tissue challenging. Therefore, ROIs with small areas were used to improve the accuracy of the placement. This study was designed as an explorative study with limited number of patients. Even though we found that further characterization of the lesions could indicate either acute pyelonephritis or alternative etiology, further studies are needed to understand the relationship with specific diagnoses. The significance of the observations and the use of the method in clinical practise also needs to be explored. 


\section{Conclusion}

Multiparametric DTI showed a diversity in renal lesions in young infants with UTI. With the use of multiparametric analyses, the lesions could be characterized further and lesion patterns could be found. Thus, multiparametric DTI has the potential to distinguish acute pyelonephritis from other renal lesions, such as permanent renal damages or dysplastic changes. As a non-invasive, non-radiating, short scan technique, multiparametric DTI is an attractive method that may provide valuable information for the evaluation of these patients in clinical practice.

\section{Abbreviations}

ADC: Apparent diffusion coefficient;; CT: Computed tomography; DMSA: 99mTc-Dimercaptosuccinic acid; DTI: Diffusion tensor imaging; DWl: Diffusion weighted imaging; FA: Fractional anisotropy; MRI: Magnetic resonance imaging; UTI: Urinary tract infections

\section{Acknowledgements}

The authors would like to thank Rune Sixt for his contribution with DMSA image analyses.

\section{Authors' contributions}

YS, KL and ES designed the study, collected and analysed the data and drafted the manuscript.

PS designed the study, collected and analysed the data. $\mathrm{HH}$ analysed the data and drafted the manuscript. SH designed the study and helped to draft the manuscript. All authors read and approved the final manuscript.

\section{Funding}

The study was supported by grants from the University of Gothenburg and Sahlgrenska University Hospital (LUA/ALF), Foundation of The Queen Silvia Children's Hospital, Gothenburg Medical Society and the Swedish Order of Freemasons in Gothenburg. The funding bodies did not have any influence on the design of the study or data collection, neither on analysis, interpretation of data or writing the manuscript. Open access funding provided by University of Gothenburg.

\section{Availability of data and materials}

The analysed data sets generated during this study are available from the corresponding author on reasonable request.

\section{Declarations}

\section{Ethics approval and consent to participate}

Written informed consent for participation in this study was obtained from the guardians of the children. The study was approved by the Swedish Ethical Review Authority in Gothenburg, Gothenburg, Sweden (Dnr 214-11)

\section{Consent for publication}

Not applicable.

\section{Competing interests}

The authors declare that they have no conflicts of interest.

\section{Author details}

'Department of Radiology, Institute of Clinical Sciences at Sahlgrenska Academy, University of Gothenburg, Gothenburg, Sweden. ²Department of Pediatrics, Institute of Clinical Sciences at Sahlgrenska Academy, University of Gothenburg, Gothenburg, Sweden. ${ }^{3}$ Department of Radiation Physics, Institute of Clinical Sciences at Sahlgrenska Academy, University of Gothenburg, Gothenburg, Sweden.
Received: 2 October 2020 Accepted: 10 June 2021

Published online: 08 October 2021

\section{References}

1. Jakobsson B, Esbjorner E, Hansson S. Minimum incidence and diagnostic rate of first urinary tract infection. Pediatrics. 1999;104(2 Pt 1):222-6.

2. Lee $\mathrm{CH}$, Yoo KH, Je BK, Kim IS, Kiefer B, Park YS, et al. Using intravoxel incoherent motion MR imaging to evaluate cortical defects in the first episode of upper urinary tract infections: preliminary results. J Magn Reson Imaging. 2014;40(3):545-51.

3. Vivier PH, Sallem A, Beurdeley M, Lim RP, Leroux J, Caudron J, et al. MRI and suspected acute pyelonephritis in children: comparison of diffusionweighted imaging with gadolinium-enhanced T1-weighted imaging. Eur Radiol. 2014;24(1):19-25.

4. De Pascale A, Piccoli GB, Priola SM, Rognone D, Consiglio V, Garetto I, et al. Diffusion-weighted magnetic resonance imaging: new perspectives in the diagnostic pathway of non-complicated acute pyelonephritis. Eur Radiol. 2013;23(11):3077-86.

5. Henninger B, Reichert M, Haneder S, Schoenberg SO, Michaely HJ. Value of diffusion-weighted MR imaging for the detection of nephritis. Sci World J. 2013;2013:348105

6. Simren Y, Stokland E, Hansson S, Sixt R, Svensson PA, Lagerstrand KM. Diffusion weighted imaging is a promising method to detect acute pyelonephritis in non-sedated free breathing infants. J Pediatr Urol. 2020; 16(3):320-5

7. Fukuda Y, Ohashi I, Hanafusa K, Nakagawa T, Ohtani S, An-naka Y, et al. Anisotropic diffusion in kidney: apparent diffusion coefficient measurements for clinical use. J Magn Reson Imaging. 2000;11(2):156-60.

8. Ries M, Jones RA, Basseau F, Moonen CT, Grenier N. Diffusion tensor MRI of the human kidney. J Magn Reson Imaging. 2001;14(1):42-9.

9. Hueper K, Gutberlet M, Rodt T, Gwinner W, Lehner F, Wacker F, et al. Diffusion tensor imaging and tractography for assessment of renal allograft dysfunction-initial results. Eur Radiol. 2011;21(11):2427-33.

10. Gaudiano C, Clementi V, Busato F, Corcioni B, Orrei M, Ferramosca E, et al. Diffusion tensor imaging and tractography of the kidneys: assessment of chronic parenchymal diseases. Eur Radiol. 2013;23(6):1678-85.

11. Notohamiprodjo M, Glaser C, Herrmann KA, Dietrich O, Attenberger UI, Reiser MF, et al. Diffusion tensor imaging of the kidney with parallel imaging: initial clinical experience. Investig Radiol. 2008;43(10):677-85.

12. Feng Q, Ma Z, Wu J, Fang W. DTI for the assessment of disease stage in patients with glomerulonephritis--correlation with renal histology. Eur Radiol. 2015;25(1):92-8.

13. Jaimes C, Darge K, Khrichenko D, Carson RH, Berman Jl. Diffusion tensor imaging and tractography of the kidney in children: feasibility and preliminary experience. Pediatr Radiol. 2014;44(1):30-41.

14. Otero HJ, Calle-Toro JS, Maya CL, Darge K, Serai SD. DTI of the kidney in children: comparison between normal kidneys and those with ureteropelvic junction (UPJ) obstruction. MAGMA. 2020;33(1):63-71.

15. Serai SD, Otero HJ, Calle-Toro JS, Berman Jl, Darge K, Hartung EA. Diffusion tensor imaging of the kidney in healthy controls and in children and young adults with autosomal recessive polycystic kidney disease. Abdom Radiol (NY). 2019:44(5):1867-72.

16. Delgado J, Berman Jl, Maya C, Carson RH, Back SJ, Darge K. Pilot study on renal magnetic resonance diffusion tensor imaging: are quantitative diffusion tensor imaging values useful in the evaluation of children with ureteropelvic junction obstruction? Pediatr Radiol. 2019;49(2):175-86.

17. Li Y, Lee MM, Worters PW, MacKenzie JD, Laszik Z, Courtier JL. Pilot study of renal diffusion tensor imaging as a correlate to histopathology in pediatric renal allografts. AJR Am J Roentgenol. 2017;208(6):1358-64.

18. Lair M, Renaux-Petel M, Hassani A, Cruypeninck Y, Vasies I, Liard A, et al. Diffusion tensor imaging in acute pyelonephritis in children. Pediatr Radiol. 2018:48(8):1081-5.

19. Piepsz A, Colarinha P, Gordon I, Hahn K, Olivier P, Roca I, et al. Guidlines on 99mTc-DMSA scintigraphy in children. 2009. https://www.eanm.org/publica tions/guidelines/paediatrics/

20. Rushton HG. The evaluation of acute pyelonephritis and renal scarring with technetium 99m-dimercaptosuccinic acid renal scintigraphy: evolving concepts and future directions. Pediatr Nephrol. 1997;11(1):108-20.

21. Majd M, Nussbaum Blask AR, Markle BM, Shalaby-Rana E, Pohl HG, Park JS, et al. Acute pyelonephritis: comparison of diagnosis with 99mTc-DMSA, 
SPECT, spiral CT, MR imaging, and power Doppler US in an experimental pig model. Radiology. 2001;218(1):101-8.

22. Feng $Q$, Fang $W$, Sun XP, Sun SH, Zhang RM, Ma ZJ. Renal clear cell carcinoma: diffusion tensor imaging diagnostic accuracy and correlations with clinical and histopathological factors. Clin Radiol. 2017;72(7):560-4.

\section{Publisher's Note}

Springer Nature remains neutral with regard to jurisdictional claims in published maps and institutional affiliations.

Ready to submit your research? Choose BMC and benefit from:

- fast, convenient online submission

- thorough peer review by experienced researchers in your field

- rapid publication on acceptance

- support for research data, including large and complex data types

- gold Open Access which fosters wider collaboration and increased citations

- maximum visibility for your research: over $100 \mathrm{M}$ website views per year

At $B M C$, research is always in progress.

Learn more biomedcentral.com/submissions 